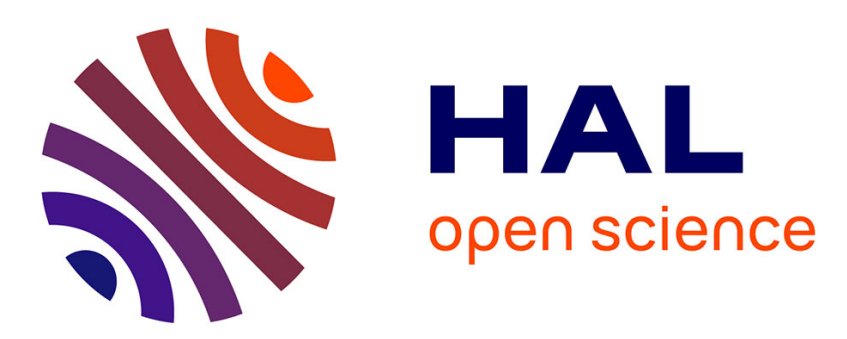

\title{
Visual Servoing from lines using a planar catadioptric system
}

Eric Marchand, Benjamin Fasquelle

\section{To cite this version:}

Eric Marchand, Benjamin Fasquelle. Visual Servoing from lines using a planar catadioptric system. IEEE/RSJ Int. Conf. on Intelligent Robots and Systems, IROS'17, Sep 2017, Vancouver, Canada. pp.2935-2940. hal-01561757

\section{HAL Id: hal-01561757 \\ https://hal.inria.fr/hal-01561757}

Submitted on 13 Jul 2017

HAL is a multi-disciplinary open access archive for the deposit and dissemination of scientific research documents, whether they are published or not. The documents may come from teaching and research institutions in France or abroad, or from public or private research centers.
L'archive ouverte pluridisciplinaire HAL, est destinée au dépôt et à la diffusion de documents scientifiques de niveau recherche, publiés ou non, émanant des établissements d'enseignement et de recherche français ou étrangers, des laboratoires publics ou privés. 


\title{
Visual Servoing from lines using a planar catadioptric system
}

\author{
Eric Marchand, Benjamin Fasquelle
}

\begin{abstract}
In this paper, we propose a complete scheme to control a mirror, using a visual servoing scheme, using lines as a set of visual features. Considering the equations of the projection of the reflection of a lines on a mirror, this paper introduces the theoretical background that allows to control the mirror using visual information. Experiments using a mirror mounted on the end-effector of a 6 d.o.f robot validate the proposed approach.
\end{abstract}

\section{INTRODUCTION}

Visual servoing [4] has proved to be a very efficient way to control robot using information provided by one or multiple cameras. Vision sensors have the main advantage to get a rich information of the robot environment. However standard perspective cameras have a limited field of view. Considering mirrors may extend the camera field of view. This is usually achieved with catadioptric camera [15] or with controlled mirror such as in the Saccade robot [9]. Few research work studied the use of mirrors in visual servoing when the relative position between the camera and mirror varies. This paper extends our prior work which dealt with the combination of visual servoing and a planar mirror [12].

Most of the literature in this area is related to the use of catadioptric camera e.g. [15][7]. In such systems, the camera and the mirror are rigidly attached and the catadioptric system is mounted on the robot. The main difference between such system and a classical visual servoing system is the projection model (central projection model) and consequently the interaction matrix. The use of planar mirrors has been considered to simulate multi-camera system. Indeed coupling a single camera with a planar mirror is is possible to obtain a stereo view. [16]. Multiple stationary planar mirrors systems for micro alignment using a visual servoing techniques was also reported in [17]. The control of the alignment process is achieved in closed loop using the projection and reflection of the the object to be aligned (optical fibers) on both mirrors. As usual with visual servoing such approach avoid precise calibration of the mirror-based stereo system. [11] report a more classical visual servoing system where the motion of the robot end-effector is controlled through its reflection in a mirror. Let us note that in [11] the mirror(s) and the camera are stationary.

Indeed, few works considered mobile mirrors or a varying mirror/camera position. Laser steering application for microsurgery is reported in [19], [2]. Whereas high speed visual servoing is concerned, some systems that consider mirrors, have been proposed to achieve high-speed gaze

Eric Marchand is with Université de Rennes 1, IRISA, Inria Rennes, France, Eric.Marchandeirisa.fr. Benjamin Fasquelle was with IRISA, France. control: Saccade mirror [10], [9]. The proposed Saccade Mirror system allows to simulate a pan/tilt camera while allowing very fast saccade and increasing the field of view of the camera. Open loop control law is considered for the tracking process. In our prior work [12], we proposed the complete modelling for the control of planar mirror using a visual servoing scheme. As far as the visual features are concerned, have been considered point. We have exhibited the interaction matrix that links the motion of a point in the image to the motion of the mirror. It has also been demonstrated that in practice only 3 mirror d.o.f. are actually controllable.

In this paper, we propose to extend [12] to the case of another visual feature: lines. Lines have already consider in visual servoing e.g. in [6][1]. Considering mirrors, lines have been considered for catadioptric system such as in [8]. In this paper we revisit the modeling proposed in [12] to adapt the proposed framework to lines. We will exhibit the interaction matrix that allow to control the mirror from the reflection of a set of lines. We will demonstrate that, in practice, only 3 d.o.f. can be controlled. Experiments using a mirror mounted on the end-effector of a 6 d.o.f robot validate the proposed approach.

\section{REFLECTION AND PROJECTION MODELING}

Let us define the mirror frame such that $\mathrm{x}$ and $\mathrm{y}$ axes are in the mirror plane $\Pi$ and $\mathrm{z}$ axis is perpendicular to the mirror (see Figure 11. The equation of the mirror in this frame is given by:

$$
{ }^{m} \mathbf{n}^{\top m} \mathbf{X}=0
$$

where ${ }^{m} \mathbf{n}=\left(\begin{array}{lll}0 & 0 & 1\end{array}\right)^{\top}$ is the normal to the mirror $\Pi$ expressed in the mirror frame.

Let us define a line $\mathcal{L}$. Expressed in the mirror frame it is parameterized by ${ }^{m} \mathbf{L}=\left({ }^{m} \mathbf{X},{ }^{m} \mathbf{u}\right)$ where ${ }^{m} \mathbf{X}$ is a point belonging to the line and ${ }^{m} \mathbf{u}$ a direction vector (see Figure 11. Let ${ }^{m} \mathbf{L}_{R}=\left({ }^{m} \mathbf{X}_{R},{ }^{m} \mathbf{u}_{R}\right)$ be the reflection of ${ }^{m} \mathbf{L}$ with respect to the mirror. ${ }^{m} \mathbf{L}_{R}$ coordinates are given by:

$$
\left\{\begin{array}{l}
{ }^{m} \mathbf{X}_{R}=\left(\mathbf{I}-2^{m} \mathbf{n}^{m} \mathbf{n}^{\top}\right)^{m} \mathbf{X} \\
{ }^{m} \mathbf{u}_{R}=\left(\mathbf{I}-2^{m} \mathbf{n}^{m} \mathbf{n}^{\top}\right)^{m} \mathbf{u}
\end{array}\right.
$$

Note that this equation is true whatever the choice of ${ }^{m} \mathbf{n}$ (subject that $\left\|^{m} \mathbf{n}\right\|=1$ ) if the origin of $\mathcal{F}_{m}$ belongs to plane $\Pi$.

Let ${ }^{c} \mathbf{X}$ be the coordinates of a point and ${ }^{c} \mathbf{u}$ the coordinates of a vector in the camera frame $\mathcal{F}_{c}$. Using classical notations for rotation and translation, we have

$$
\left\{\begin{array}{l}
{ }^{c} \mathbf{X}={ }^{c} \mathbf{R}_{m}{ }^{m} \mathbf{X}+{ }^{c} \mathbf{t}_{m} \\
{ }^{c} \mathbf{u}={ }^{c} \mathbf{R}_{m}{ }^{m} \mathbf{u} .
\end{array}\right.
$$


Let

$$
\mathbf{n}^{\top c} \mathbf{X}=d
$$

be the equation of the mirror plane in the camera frame where $\mathbf{n}$ is the unit vector that represents the normal to the plane in the camera frame and $d$ is the orthogonal distance between the camera and the plane (see Figure 11). We thus have $\mathbf{n}={ }^{c} \mathbf{R}_{m}{ }^{m} \mathbf{n}$ and ${ }^{c} \mathbf{t}_{m}=d \mathbf{n}$.

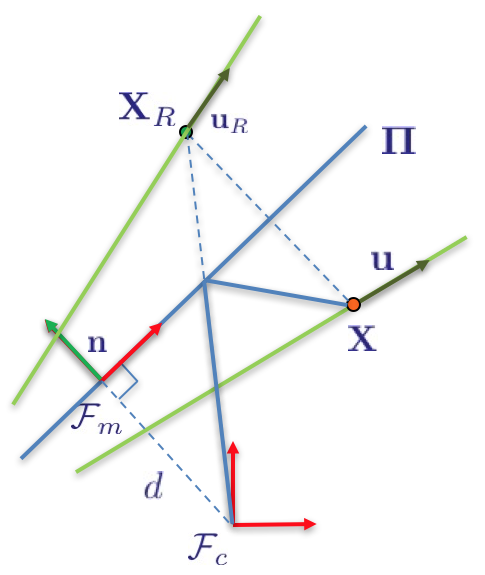

Fig. 1. Reflection and projection with a planar mirror

The coordinates of the reflection ${ }^{c} \mathbf{L}_{R}$ of ${ }^{c} \mathbf{L}$ wrt. the mirror is given by [16] (see proof in Appendix A):

$$
\left\{\begin{array}{l}
{ }^{c} \mathbf{X}_{R}=\left(\mathbf{I}-2 \mathbf{n} \mathbf{n}^{T}\right)^{c} \mathbf{X}+2 d \mathbf{n} \\
{ }^{c} \mathbf{u}_{R}=\left(\mathbf{I}-2 \mathbf{n} \mathbf{n}^{T}\right)^{c} \mathbf{u}
\end{array}\right.
$$

Finally, whereas the projection of the line in the image is considered, we choose the representation $\mathbf{l}=(\rho, \theta)^{\top}$ (see Figure 2) and the equation of the projection of a line $\mathcal{L}$ is given by:

$$
x \cos (\theta)+y \sin (\theta)-\rho=0
$$

This representation is minimal and the ambiguity $((\rho, \theta \pm$ $2 k \pi)$ and $(-\rho, \theta \pm(2 k+1) \pi)$ represent the same line) can be overcomed by considering that $\theta \in\left[-\frac{\pi}{2}, \frac{\pi}{2}\right]$ which will ensure a continuous control law. Finally, the projection $\mathbf{l}=$ $(\rho, \theta)^{\top}$ of a straight line $\left({ }^{c} \mathbf{X}_{R},{ }^{c} \mathbf{u}_{R}\right)$ is given by:

$$
\left\{\begin{array}{l}
\theta=\arctan \left(\frac{u_{y}}{u_{x}}\right) \\
\rho=\frac{X_{R}}{Z_{R}} \cos (\theta)+\frac{Y_{R}}{Z_{R}} \sin (\theta)
\end{array}\right.
$$

where ${ }^{c} \mathbf{X}_{R}=\left(X_{R}, Y_{R}, Z_{R}\right)^{\top}$ and ${ }^{c} \mathbf{u}_{R}=\left(u_{x}, u_{y}, u_{z}\right)^{\top}$

\section{VISUAL SERVOING THROUGH MIRROR}

Let us consider a basic visual servoing scheme that is specified by observing a set of $2 \mathrm{D}$ lines $\mathrm{l}_{i}$ at given positions in the image $\mathbf{l}_{i}^{*}$. Thus we want to minimize the norm of the error e defined by:

$$
\mathbf{e}=\left(\begin{array}{c}
\vdots \\
\mathbf{l}_{i}-\mathbf{l}_{i}^{*} \\
\vdots
\end{array}\right)
$$

In this paper we will only consider the case of a controlled mirror mounted on the robot effector (see Figure 3). Other

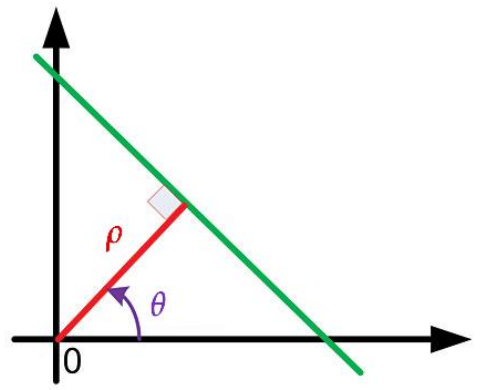

Fig. 2. Representation $(\rho, \theta)$ of a line in the image space.

configuration of the triplet camera/robot/mirror can be found and have been explored in the case of points in [12].

Thus, one wants to observe a target at a given position in the image through its reflection in the mirror. To design the control law one has to determine the Jacobian $\mathbf{J}_{\mathbf{l}}$ that links the motion of a set of lines in the image to the motion of the end effector (on which the mirror is attached) in the joint space, that is:

$$
\dot{\mathrm{i}}=\mathbf{J}_{\mathbf{l}} \dot{\mathbf{q}}
$$

where $\dot{\mathbf{q}}$ is the robot joint velocity. In that case, considering that we want an exponential decrease of $\mathbf{e}$, the control law is given by:

$$
\dot{\mathbf{q}}=-\lambda \mathbf{J}_{1}^{+} \mathbf{e}
$$

To determine $\mathbf{J}_{1}$, one has to derive the relation (the interaction matrix [4]) that links the motion $\mathrm{i}$ of a line $\mathcal{L}$ to the motion of the mirror. This is the purpose of the next section.

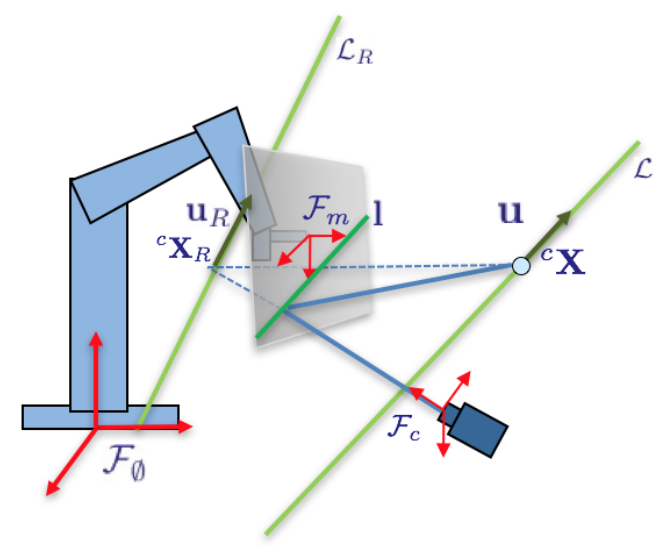

Fig. 3. System configuration

\section{Controlling The MirRor POSITION}

\section{A. Definition of the interaction matrix and of the control law}

This section is devoted to the definition of the interaction matrix that links the motion $\mathrm{i}$ of a line $\mathcal{L}$ in the image to the motion of the mirror. That is we seek the matrix $\mathbf{L}_{\mathbf{l}}$ defines such that:

$$
\mathrm{i}=\mathbf{L}_{\mathbf{l}}{ }^{c} \mathbf{v}_{m}
$$


where ${ }^{c} \mathbf{v}_{m}$ is the mirror motion expressed in the camera frame $\left({ }^{c} \mathbf{v}_{m}=\left({ }^{c} \boldsymbol{v}_{m},{ }^{c} \boldsymbol{\omega}_{m}\right)^{\top}\right.$ where ${ }^{c} \boldsymbol{v}_{m}$ is the mirror translational velocity and ${ }^{c} \boldsymbol{\omega}_{m}$ is the mirror rotational velocity).

Equation (11) can be rewritten as:

$$
\mathrm{i}=\mathbf{L}\left(\begin{array}{c}
{ }^{c} \dot{\mathbf{X}}_{R} \\
{ }^{c} \dot{\mathbf{u}}_{R}
\end{array}\right)
$$

where $\left({ }^{c} \dot{\mathbf{X}}_{R},{ }^{c} \dot{\mathbf{u}}_{R}\right)^{\top}$ represents the motion of the 3D line wrt. to the mirror motion and $\mathbf{L}$ links the motion of the line projection to the motion of the 3D line.

Let us first examine the derivation of the interaction matrix related to $\left({ }^{c} \mathbf{X}_{R},{ }^{c} \mathbf{u}_{R}\right)^{\top}$. It is given by:

$$
\left(\begin{array}{c}
{ }^{c} \dot{\mathbf{X}}_{R} \\
{ }^{c} \dot{\mathbf{u}}_{R}
\end{array}\right)=\left(\begin{array}{cc}
\mathbf{L}_{\boldsymbol{v}} & \mathbf{L}_{\boldsymbol{\omega} \mathbf{X}} \\
\mathbf{0}_{3 \times 3} & \mathbf{L}_{\boldsymbol{\omega} \mathbf{u}}
\end{array}\right)\left(\begin{array}{c}
{ }^{c} \boldsymbol{v}_{m} \\
{ }^{c} \boldsymbol{\omega}_{m}
\end{array}\right)
$$

with

$$
\begin{aligned}
\mathbf{L}_{\boldsymbol{v}} & =-2 \mathbf{n} \mathbf{n}^{T} \\
\mathbf{L}_{\boldsymbol{\omega} \mathbf{X}} & =-2\left(d[\mathbf{n}]_{\times}+\left[{ }^{c} \mathbf{X}_{R}\right]_{\times}[\mathbf{n}]_{\times}^{2}\right) \\
\mathbf{L}_{\boldsymbol{\omega} \mathbf{u}} & =-2\left[{ }^{c} \mathbf{u}_{R}\right]_{\times}[\mathbf{n}]_{\times}^{2}
\end{aligned}
$$

where $[\mathbf{a}]_{\times}$denotes the skew symmetric matrix associated to the vector $\mathbf{a}$.

Proof: Indeed, as seen in [12], it is possible to show that:

$$
{ }^{c} \dot{\mathbf{X}}_{R}=2 \mathbf{n} \dot{d}-2 \dot{\mathbf{n}} d+2\left(\dot{\mathbf{n}} \mathbf{n}^{\top}-\mathbf{n} \dot{\mathbf{n}}^{\top}\right)^{c} \mathbf{X}_{R}
$$

The interaction matrix related to a plane given by (4) is given by [20][18]:

$$
\left(\begin{array}{c}
\dot{\mathbf{n}} \\
\dot{d}
\end{array}\right)=\left(\begin{array}{cc}
\mathbf{0}_{3 \times 3} & {[\mathbf{n}]_{\times}} \\
-\mathbf{n}^{\top} & \mathbf{0}_{1 \times 3}
\end{array}\right)\left(\begin{array}{c}
{ }^{c} \boldsymbol{v}_{m} \\
{ }^{c} \boldsymbol{\omega}_{m}
\end{array}\right)
$$

When considering $\mathbf{L}_{\boldsymbol{v}}$, according to equation (18) we thus have

$$
2 \mathbf{n} \dot{d}=2 \mathbf{n}\left(-\mathbf{n}^{\top} \mathbf{0}_{1 \times 3}\right)\left(\begin{array}{c}
{ }^{c} \boldsymbol{v}_{m} \\
{ }^{c} \boldsymbol{\omega}_{m}
\end{array}\right)=-2 \mathbf{n} \mathbf{n}^{\top c} \boldsymbol{v}_{m}
$$

As far as $\mathbf{L}_{\boldsymbol{\omega} \mathbf{X}}$ is concerned

$$
2 \dot{\mathbf{n}} d=2 d\left(\mathbf{0}_{3 \times 3}[\mathbf{n}]_{\times}\right)\left(\begin{array}{c}
{ }^{c} \boldsymbol{v}_{m} \\
{ }^{c} \boldsymbol{\omega}_{m}
\end{array}\right)=2 d[\mathbf{n}]_{\times}{ }^{c} \boldsymbol{\omega}_{m}
$$

and, $\dot{\mathbf{n}}^{\top}-\mathbf{n} \dot{\mathbf{n}}^{\top}$ being an anti-symmetric matrix, it can be demonstrated that

$$
\begin{aligned}
\dot{\mathbf{n}}^{\top}-\mathbf{n} \dot{\mathbf{n}}^{\top} & =\left[[\mathbf{n}]_{\times} \dot{\mathbf{n}}\right]_{\times}=\left[[\mathbf{n}]_{\times}[\mathbf{n}]_{\times}{ }^{c} \boldsymbol{\omega}_{m}\right]_{\times} \\
& =\left[[\mathbf{n}]_{\times}^{2 c} \boldsymbol{\omega}_{m}\right]_{\times}
\end{aligned}
$$

leading to

$$
\begin{aligned}
\left(\dot{\mathbf{n}} \mathbf{n}^{\top}-\mathbf{n} \dot{\mathbf{n}}^{\top}\right)^{c} \mathbf{X}_{R} & =\left[[\mathbf{n}]_{\times}^{2 c}{ }^{c} \boldsymbol{\omega}_{m}\right]_{\times}{ }^{c} \mathbf{X}_{R} \\
& =-\left[{ }^{c} \mathbf{X}_{R}\right]_{\times}[\mathbf{n}]_{\times}^{2 c}{ }^{c} \boldsymbol{\omega}_{m}
\end{aligned}
$$

When dealing with $\mathbf{L}_{\boldsymbol{\omega} \mathbf{u}}$ in 16 we can prove that:

$$
{ }^{c} \dot{\mathbf{u}}_{R}=2\left(\dot{\mathbf{n}} \mathbf{n}^{\top}-\mathbf{n} \dot{\mathbf{n}}^{\top}\right)^{c} \mathbf{u}_{R}
$$

Considering (24) we have indeed proved (16) since (as for (24))

$$
\left(\dot{\mathbf{n}} \mathbf{n}^{\top}-\mathbf{n} \dot{\mathbf{n}}^{\top}\right)^{c} \dot{\mathbf{u}}_{R}=-\left[{ }^{c} \dot{\mathbf{u}}_{R}\right]_{\times}[\mathbf{n}]_{\times}^{2}{ }^{c} \boldsymbol{\omega}_{m}
$$

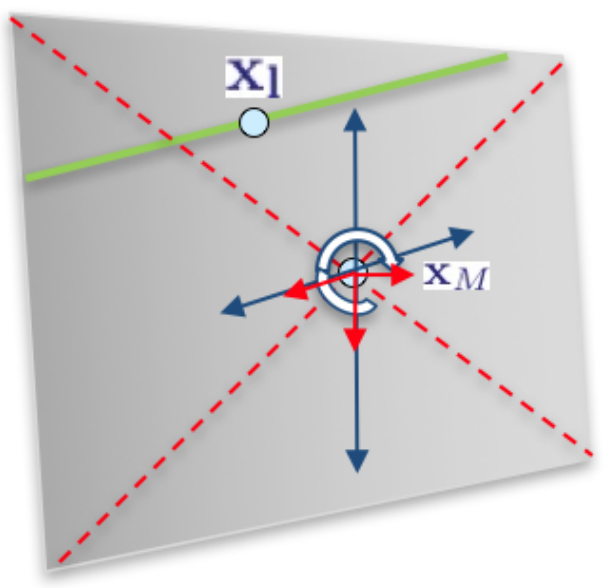

Fig. 4. Invariance of object reflection to in-plane mirror motions (in blue) and how to use the remaining d.o.f by minimizing the error $\mathbf{x}_{\mathbf{M}}-\mathbf{x}_{\mathbf{l}}$

Whereas $\mathbf{L}$ in equation $(12)$ is concerned, it is given by:

$$
\mathbf{L}=\left(\begin{array}{cc}
a \cos (\theta) & 0 \\
a \sin (\theta) & \\
-a^{2}\left(X_{R} \cos (\theta)+Y_{R} \sin (\theta)\right) & 0 \\
-a b\left(Y_{R} \cos (\theta)-X_{R} \sin (\theta)\right) & -b \\
a c\left(Y_{R} \cos (\theta)-X_{R} \sin (\theta)\right) & c \\
0 & 0
\end{array}\right)^{\top}
$$

with $a=\frac{1}{Z_{R}}, b=\frac{u_{y}}{u_{x}^{2}+u_{y}^{2}}$ and $c=\frac{u_{x}}{u_{x}^{2}+u_{y}^{2}}$. Leading to equation (25). Note that besides $Z_{R}$ and $u_{z}$ most of the parameters involved in $\mathbf{L}$ are easy to infer from the $2 \mathrm{D}$ information.

The final interaction matrix defined in (11) is then given by

$$
\mathbf{L}_{\mathbf{l}}=\mathbf{L}\left(\begin{array}{cc}
\mathbf{L}_{\boldsymbol{v}} & \mathbf{L}_{\boldsymbol{\omega}} \mathbf{X} \\
\mathbf{0}_{3 \times 3} & \mathbf{L}_{\boldsymbol{\omega} \mathbf{u}}
\end{array}\right)
$$

Finally, to control the mirror motion in the mirror frame $\mathcal{F}_{m}$, we have

$$
\left(\begin{array}{c}
\dot{\rho} \\
\dot{\theta}
\end{array}\right)=\mathbf{L}_{\mathbf{l}}^{c} \mathbf{V}_{m}\left(\begin{array}{c}
m \\
\mathbf{v}_{m} \\
{ }^{m} \omega_{m}
\end{array}\right)
$$

with ${ }^{c} \mathbf{V}_{m}$ the spatial motion transform matrix from the camera frame to the mirror frame [4] .We get the Jacobian $\mathbf{J}_{\mathbf{1}}$ :

$$
\mathbf{J}_{\mathbf{l}}=-\mathbf{L}_{\mathbf{l}}{ }^{c} \mathbf{V}_{m}{ }^{m} \mathbf{V}_{e}{ }^{e} \mathbf{J}(\mathbf{q})
$$

B. Actual number of controllable d.o.f and how to use the remaining ones

Using such a configuration, it is easy to see that motions in the image plane do not modify the position of the line (l) in the image. When the system is perfectly calibrated first, second and sixth column of $\mathbf{L}_{\mathbf{l}}^{c} \mathbf{V}_{m}$ are equal to zero. That is:

$$
\operatorname{rank} \mathbf{L}_{\mathbf{l}}^{c} \mathbf{V}_{m}=3 \text {. }
$$

Proof: Let us first develop $\mathbf{L}_{\mathbf{l}}{ }^{c} \mathbf{V}_{m}$. We have:

$$
{ }^{c} \mathbf{V}_{m}=\left(\begin{array}{cc}
{ }^{c} \mathbf{R}_{m} & d[\mathbf{n}]_{\times}{ }^{c} \mathbf{R}_{m} \\
\mathbf{O}_{3 \times 3} & { }^{c} \mathbf{R}_{m}
\end{array}\right)
$$


Leading to (see [12])

$$
\mathbf{L}_{\mathbf{l}}{ }^{c} \mathbf{V}_{m}=\mathbf{L}\left(\begin{array}{cc}
\mathbf{L}_{\boldsymbol{v}}{ }^{c} \mathbf{R}_{m} & \mathbf{L}_{\omega \mathbf{X}}{ }^{c} \mathbf{R}_{m} \\
\mathbf{O}_{3 \times 3} & \mathbf{L}_{\omega \mathbf{u}}{ }^{c} \mathbf{R}_{m}
\end{array}\right)
$$

It remain to show that the two first column of $\mathbf{L}_{\boldsymbol{v}}{ }^{c} \mathbf{R}_{m}$, and the thirst column of $\mathbf{L}_{\boldsymbol{\omega} \mathbf{X}}{ }^{c} \mathbf{R}_{m}$ as well as of $\mathbf{L}_{\boldsymbol{\omega} \mathbf{u}}{ }^{c} \mathbf{R}_{m}$ are equal to zero. On one hand, we have [12]:

$$
\mathbf{L}_{\boldsymbol{v}}{ }^{c} \mathbf{R}_{m}=-2 \mathbf{n}^{m} \mathbf{n}^{\top}
$$

Since ${ }^{m} \mathbf{n}^{\top}=\left(\begin{array}{lll}0 & 0 & 1\end{array}\right)^{\top}$, denoting $\mathbf{n}^{\top}=\left(\begin{array}{lll}n_{1} & n_{2} & n_{3}\end{array}\right)^{\top}$, on a:

$$
\mathbf{L}_{\boldsymbol{v}}{ }^{c} \mathbf{R}_{m}=-2\left(\begin{array}{ccc}
0 & 0 & n_{1} \\
0 & 0 & n_{2} \\
0 & 0 & n_{3}
\end{array}\right)
$$

On the other hand we have:

$$
-\frac{1}{2} \mathbf{L}_{\omega \mathbf{X}}{ }^{c} \mathbf{R}_{m}=d^{c} \mathbf{R}_{m}\left[{ }^{m} \mathbf{n}\right]_{\times}+\left[{ }^{c} \mathbf{X}_{R}\right]_{\times}{ }^{c} \mathbf{R}_{m}\left[{ }^{m} \mathbf{n}\right]_{\times}^{2}
$$

Expending ${ }^{m} \mathbf{n}^{\top}=\left(\begin{array}{lll}0 & 0 & 1\end{array}\right)^{\top}$ it is immediate to see that the last column of $\mathbf{L}_{\omega \mathbf{X}}{ }^{c} \mathbf{R}_{m}$ is $(0,0,0)^{\top}$. We also have:

$$
\begin{aligned}
\mathbf{L}_{\omega \mathbf{u}}{ }^{c} \mathbf{R}_{m} & =-2\left[{ }^{c} \mathbf{u}_{R}\right]_{\times}[\mathbf{n}]_{\times}^{2}{ }^{c} \mathbf{R}_{m} \\
& =-2\left[{ }^{c} \mathbf{u}_{R}\right]_{\times}{ }^{c} \mathbf{R}_{m}\left[{ }^{m} \mathbf{n}\right]_{\times}^{2}
\end{aligned}
$$

Again, expending ${ }^{m} \mathbf{n}^{\top}=\left(\begin{array}{lll}0 & 0 & 1\end{array}\right)^{\top}$ it is immediate to see that the last column of $\mathbf{L}_{\omega \mathbf{u}}{ }^{c} \mathbf{R}_{m}$ is $(0,0,0)^{\top}$.

We thus have $\operatorname{rank} \mathbf{L}_{\mathbf{l}}{ }^{c} \mathbf{V}_{m}=3$

Since only 3 d.o.f are actually controlled it is then possible to use the 3 remaining ones considering the redundancy framework [4], [5] to achieve other task. This is of interest here since the mirror is not of infinite size. Indeed since only orientation and depth of the mirror can be controlled, the target may "slip" out the mirror. It is then usually necessary to consider the remaining d.o.f to maintain the lines within the mirror during the visual servoing task.

Let us consider here that we control the robot in the mirror frame. As stated above, since rank $\mathbf{J}_{\mathbf{1}}=3$, the control law can be expressed by:

$$
\mathbf{v}_{m}=-\lambda \mathbf{J}_{\mathbf{1}}^{+} \mathbf{e}+\mathbf{P}_{\mathbf{l}} \mathbf{J}_{\mathbf{e}_{\mathbf{2}}}^{+} \mathbf{e}_{\mathbf{2}}
$$

with $\mathbf{P}_{1}=\mathbf{I}-\mathbf{J}_{1}^{+} \mathbf{J}_{1}$ is a projection operator which ensure that $\mathbf{e}_{2}$ has no effect on the main task $\mathbf{e}$. Our goal is to maintain the center of the line in center of the mirror. When 2 lines are concerned the intersection of the two lines is maintained at the center of the mirror. When more lines are concerned, other strategies can be foreseen. If $\mathbf{x}_{M}$ be the center of gravity of the mirror and $\mathrm{x}_{1}$ the center of gravity of the segment in the image (see Figure 4). The cost function $\mathbf{e}_{2}$ is then defined as

$$
\mathbf{e}_{2}=\beta\left(\mathbf{x}_{M}-\mathbf{x}_{\mathbf{l}}\right)
$$

where $\beta$ is a positive scalar. Note that $\mathbf{x}_{\mathbf{l}}$, being computed from the reflection of the line is invariant, by definition, to the motion of the mirror involved by this secondary task. $\mathbf{x}$ being invariant, the Jacobian $\mathbf{J}_{\mathbf{e}_{2}}$ only links the motion of $\mathbf{x}_{M}$ to the motion of the mirror. It is given by the classical interaction matrix related to the point $\mathbf{x}_{M}$ [4]. This process is similar to the one considered in [12] to maintain points on the mirror.

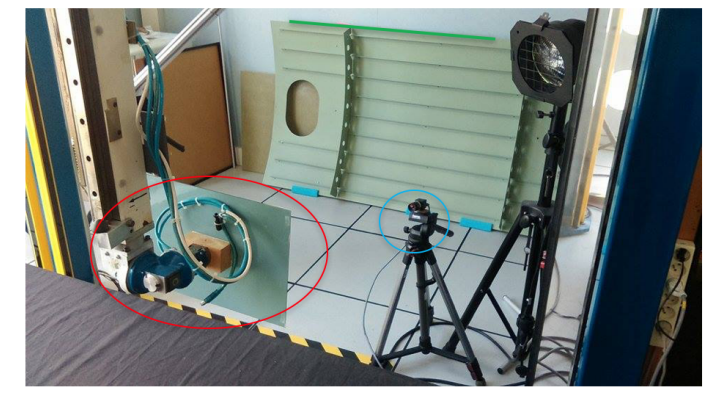

Fig. 5. Experimental setup: the mirror (red circle) is mounted on the robot end effector; the camera (blue circles) observes the target (green line) as its reflection in the mirror.

\section{EXPERIMENTAL RESULTS}

We validated the presented approach with experiments carried out on a 6 dof robot.

\section{A. Experimental setup}

Figure 5 shows the experimental setup. The mirror is mounted on the end-effector of a 6 d.o.f gantry robot. The camera (which is fully calibrated) is static and looks at the mirror and (thanks to the mirror reflection) to the target (see Figure 7). The implementation has be done thanks to the ViSP software [13].

The relative position between the camera and the robot base frame is computed thanks to the localization of the mirror wrt the camera using a non-linear pose estimation method [14] using four points located on the mirror corners (see Figure 7). This is done to compute the constant matrix ${ }^{c} \mathbf{V}_{m}$ (see (28)). It is also used to compute the center of the mirror. This information is used within the secondary task to avoid the line to slide out of the mirror as explained in section IV-B

\section{B. Line tracking}

We have considered a fast real-time tracking process for line tracking. We have considered a local approach that allows to match moving contours. We decided to use the ME (moving edges) algorithm [3] adapted to the tracking of parametric curves in general and in our case to line. One of the advantages of the method is that it does not require any edge extraction, furthermore it can be implemented with convolution efficiency and can therefore ensure a real-time computation. As we want an algorithm that is fast, reliable, robust to partial occlusions and to false matches, we decided to track only a list $L^{t}$ of pixels along the considered edge and then to determine, by a robust least square approach, the equation of the support primitive that fits these data.

After the initialization of the tracking process a the pixels list $L(t)$ is available. We estimate the normal direction to the line given by the $\theta$ parameters. The local tracking process consists in searching for the correspondent $\mathbf{x}(t+1)$ in image $I(t+1)$ of each pixel $\mathbf{x}(t) \in L^{t}$. We determine a $1 \mathrm{D}$ search area $\mathbf{x}^{j}(t+1), j \in[-J, J]$ in the direction normal to the line. For each pixel $\mathbf{x}(t)$ of the list $L^{t}$, and for each position $\mathbf{x}^{j}$ lying in the direction normal to the line we compute 


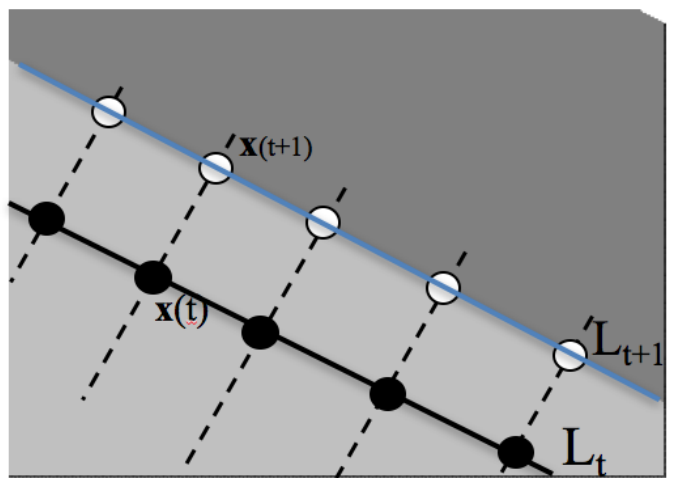

Fig. 6. Line tracking overview

the matching criterion corresponding to a log-likelihood ratio $\zeta^{j}$. This is nothing but the absolute sum of the convolution values computed at $\mathbf{x}_{i}$ and $\mathbf{y}^{j}$ using a pre-determined mask $M_{\theta}$ function of the orientation of the contour [3]. New position $\widehat{\mathbf{x}}(t+1)$ is given by:

$$
\widehat{\mathbf{x}}(t+1)=\arg \max _{\mathbf{x}^{j}(t+1), j \in[-J, J]} \zeta^{j}
$$

with

$$
\zeta^{j}=\left|I(\mathbf{x}(t)) * M_{\theta}+I\left(\mathbf{x}^{j}(t+1)\right) * M_{\theta}\right|
$$

providing that the value $\zeta^{j^{*}}$ is greater than a threshold $\lambda$. Then pixel $\mathrm{x}^{t+1}$ is stored in $L^{t+1}$. A new list of pixels is obtained. It is possible to interpret this criterion by: we look for a strong gradient along the normal to the line $I\left(\mathrm{x}^{j}(t+\right.$ 1)) $* M_{\theta}$ and want this contour to be similar to the one in the previous image $I(\mathbf{x}(t)) * M_{\theta}$.

Finally, given the list $L^{t+1}$ the new parameters of the feature are computed using an robust least squares technique (Iterative reweighted least square) leading to estimation of the parameter $\rho$ and $\theta$ of the line. A points insertion processes either in the middle of the segment, to deal with partial occlusions or miss-tracking, and at the extremities of the segment to deal with sliding movements has been introduced in the tracking method. This method is implemented in ViSP [13].

\section{Servo on one line}

This first experiments depicts a basic visual servoing task on a line. The reflection of the target (red line) has to be seen on the green line (see Figure 7 top) using the control law given by (28). As expected, 3 d.o.f are actually involved to achieve the task (see Figure 6c). In this experiment mainly the orientation of the mirror is involved but a (very) small motion along the $\mathrm{Z}$ axis can be also observed (see Figure 6d). The depth involved in (25) has been set to $2 \mathrm{~m}$, which roughly correspond to the double of the distance between the camera and the mirror. The errors feature an exponential decay (see Figure 6a), which shows the robustness of the control scheme wrt. coarse approximation of the depth and to noisy measurements. Note that measurement (especially for the $\theta$ parameters) are indeed quite noisy due

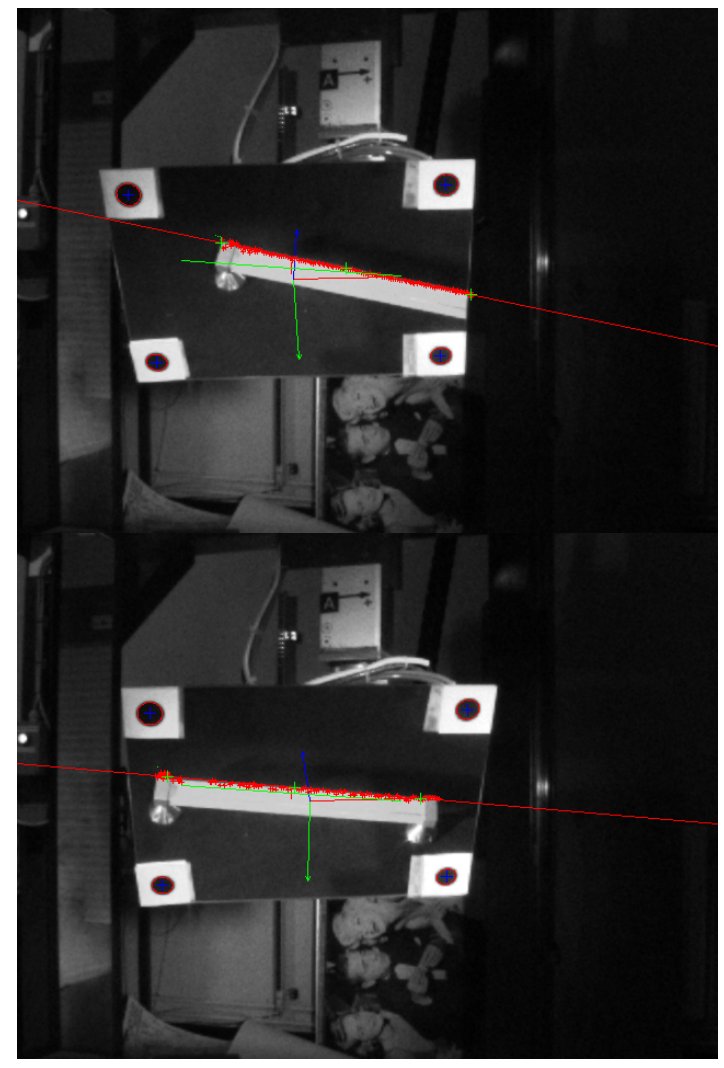

Fig. 7. Servo on one line: initial position (top) and desired (and final) position (bottom).

to the low contrast of the line in the image, the small size of the line and the poor quality of the mirror.

\section{Servo on 2 lines}

In this case, we consider a target made of 2 lines. The initial and final (desired) image are shown on Figure 9. In this case 3 d.o.f are fully controlled. Here not only the orientation of the mirror is involved but its depth (in the camera frame) as well. Furthermore, due to the mirror limited size, it is necessary to use the secondary task defined in Section IV-B in order to avoid the target moving out of the mirror. We see in that case that, finally, all the mirror d.o.f are used (see Figure 10 bottom-right). This can be clearly seen on Figure 10 (bottom right), which depicts mirror orientation and depth. The mirror converges toward a desired pose (up to a translation in the mirror plane and a rotation around its normal). The depth ${ }^{c} Z_{R}$ involved in (25) is coarsely set to $2 \mathrm{~m}$. It can be seen on Figure 10 than the camera moves backward $20 \mathrm{~cm}$ to reach a desired position. As stated the measure of the mirror parameters (Figure 10 (bottom right)) are computed thanks to a pose estimation process.

\section{CONCLUSION}

In this paper we extended the work presented in [12] related visual servoing through mirror reflection and proposed the theoretical background necessary to derive the control law when considering lines as visual features. We exhibit the analytical form of the interaction matrix and the resulting 

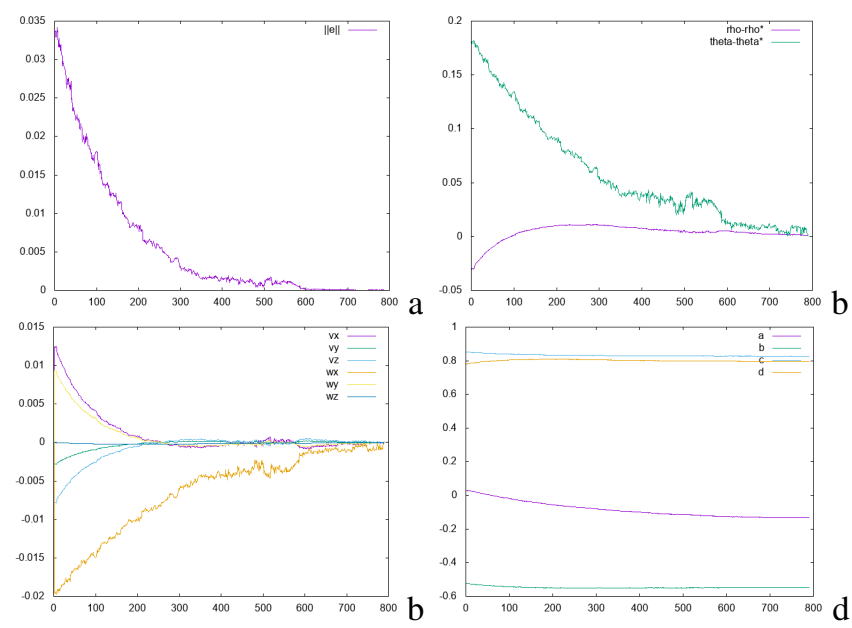

Fig. 8. Servo on one line with the use of a secondary task as proposed in section IV-B (a) $\left\|\mathbf{l}-\mathbf{l}^{*}\right\|$ (b) error $\mathbf{l}-\mathbf{l}^{*}$, (c) mirror velocities $\left({ }^{m} \boldsymbol{v}_{m},{ }^{m} \boldsymbol{\omega}_{m}\right)$ (d) plane equation. Note that in that case all the d.o.f. are used to maintain the target in the mirror.
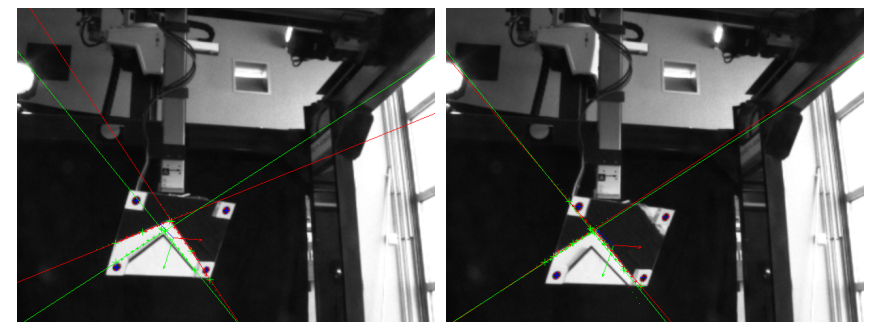

Fig. 9. Servo on two lines with the use of a secondary task as proposed in section IV-B initial position (left) and desired (and final) position (right).
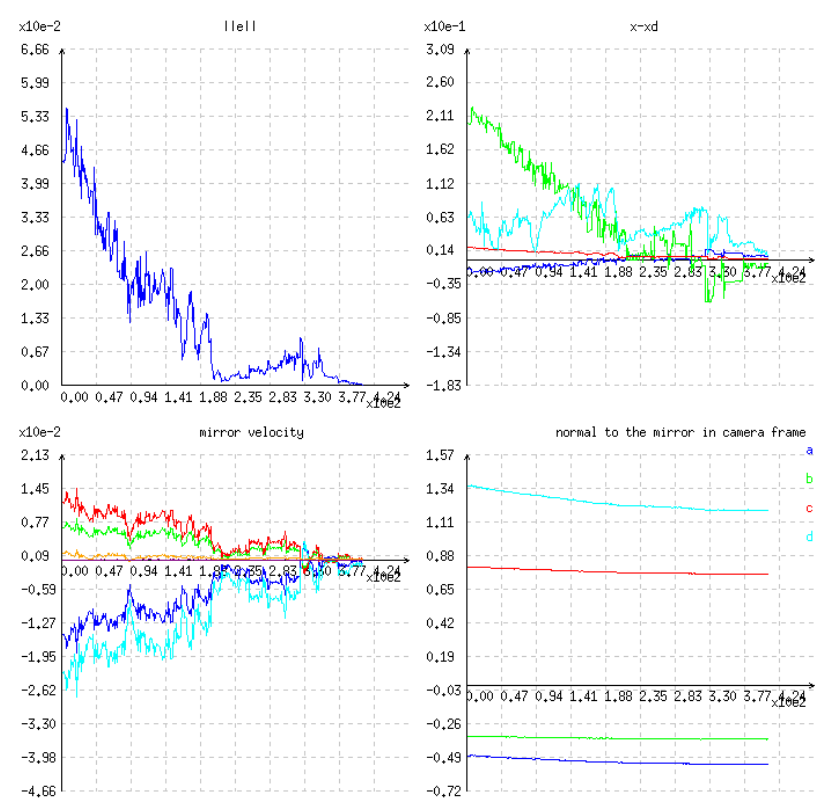

Fig. 10. Servo on two lines with the use of a secondary task as proposed in section IV-B (top left) $\left\|\mathbf{l}-\mathbf{l}^{*}\right\|$ (top right) error $\mathbf{l}_{i}-\mathbf{l}_{i}^{*}, i=1 . .2$, (bottom left) mirror velocities $\left({ }^{m} \boldsymbol{v}_{m},{ }^{m} \boldsymbol{\omega}_{m}\right)^{\top}$ (bottom right) plane equation. Note that in that case all the d.o.f. are used to maintain the target in the mirror. control law. Experiments has been achieved with a Gantry robot to show the validity of our approach in the case of controlled mirror.

\section{REFERENCES}

[1] N. Andreff, B. Espiau, and R. Horaud. Visual servoing from lines. Int. Journal of Robotics Research, 21(8):669-699, August 2002.

[2] N. Andreff and B. Tamadazte. Laser steering using virtual trifocal visual servoing. The Int. Journal of Robotics Research, 35(6):672694, 2016.

[3] P. Bouthemy. A maximum likelihood framework for determining moving edges. IEEE Trans. on Pattern Analysis and Machine Intelligence, 11(5):499-511, May 1989.

[4] F. Chaumette, S. Hutchinson, and P. Corke. Visual Servoing. In O. Khatib B. Siciliano, editor, Handbook of Robotics, 2nd edition, pages 841-866. Springer, 2016.

[5] F. Chaumette and E. Marchand. A new redundancy-based iterative scheme for avoiding joint limits: application to visual servoing. In IEEE Int. Conf. on Robotics and Automation, ICRA'O0, volume 2, pages 1720-1725, San Francisco, USA, April 2000.

[6] B. Espiau, F. Chaumette, and P. Rives. A new approach to visual servoing in robotics. IEEE Trans. on Robotics and Automation, 8(3):313-326, June 1992

[7] H. Hadj Abdelkader, Y. Mezouar, N. Andreff, and P. Martinet. Imagebased control of mobile robot with central catadioptric cameras. In IEEE Int. Conf. on Robotics and Automation, ICRA'05, pages 35223527, Barcelona, Spain, April 2005.

[8] H. Hadj Abdelkader, Y. Mezouar, N. Andreff, and P. Martinet. Omnidirectional visual servoing from polar lines. In IEEE Int. Conf. on Robotics and Automation, ICRA'06, pages 2385-2390, Orlando, Fl., May 2006.

[9] K. Iida and H. Oku. Saccade mirror 3: High-speed gaze controller with ultra wide gaze control range using triple rotational mirrors. In IEEE Int. Conf. on Robotics and Automation, ICRA'16, pages 624629, Stockholm, Sweden, May 2016.

[10] K. K. Okumura, H. Oku, and M. Ishikawa. High-speed gaze controller for millisecond-order pan/tilt camera. In IEEE Int. Conf. on Robotic and Automation, ICRA'11, pages 6186-6191, May 2011.

[11] C. Kulpate, R. Paranjape, and M. Mehrandezh. Precise 3d positioning of a robotic arm using a single camera and a flat mirror. Int. Journal of Optomechatronics, 2(3):205-232, 2008.

[12] E. Marchand and F. Chaumette. Visual servoing through mirror reflection. In IEEE Int. Conf. on Robotics and Automation, ICRA'17, Singapore, May 2017.

[13] E. Marchand, F. Spindler, and F. Chaumette. ViSP for visual servoing: a generic software platform with a wide class of robot control skills IEEE Robotics and Automation Magazine, 12(4):40-52, December 2005.

[14] E. Marchand, H. Uchiyama, and F. Spindler. Pose estimation for augmented reality: a hands-on survey. IEEE Trans. on Visualization and Computer Graphics, 22(12):2633-2651, December 2016.

[15] G.-L. Mariottini and D. Prattichizzo. Image-based visual servoing with central catadioptric cameras. The International Journal of Robotics Research, 27(1):41-56, January 2008.

[16] G.-L. Mariottini, S. Scheggi, F. Morbidi, and D. Prattichizzo. Planar catadioptric stereo: single and multi-view geometry for calibration and localization. In IEEE Int. Conf. on Robotics and Automation, ICRA'09, pages 1510-1515, Kobe, Japan, 2009.

[17] M. Mikawa, K. Yoshida, M. Kubota, and T. Morimitsu. Visual servoing for micro mass axis alignment device. In IEEE/RSJ Int. Conf. on Intelligent Robots and Systems, IROS'96, pages 1091-1096, Osaka, Japan, November 1996.

[18] J. Pagès, C. Collewet, F. Chaumette, and J. Salvi. Visual servoing by means of structured light for plane-to-plane positioning. Technical Report 5579, INRIA, May 2005.

[19] B. Tamadazte and N. Andreff. Weakly calibrated stereoscopic visual servoing for laser steering: Application to phonomicrosurgery. In IEEE/RSJ Int. Conf. on Intelligent Robots and Systems, IROS'14, pages 743-748, Chicago, IL, 2014.

[20] J.-P. Urban, G. Motyl, and J. Gallice. Real-time visual servoing using controlled illumination. The International Journal of Robotics Research, 13(1):93-100, February 1994. 